\title{
Ultra-compact structures in galactic masers observed in the RadioAstron project
}

\author{
N. N. Shakhvorostova* ${ }^{a, b}$, A. M. Sobolev ${ }^{b}$, A. V. Alakoz ${ }^{a}$, J. M. Moran ${ }^{c}$, H. Imai ${ }^{d}$, \\ V. Yu. Avdeev ${ }^{a}$ \\ ${ }^{a}$ Astro Space Center, Lebedev Physical Institute, Russian Academy of Sciences, Moscow, Russia \\ ${ }^{b}$ Astronomical Observatory, Institute for Natural Sciences and Mathematics, Ural Federal \\ University, Ekaterinburg, Russia \\ ${ }^{c}$ Harvard-Smithsonian Center for Astrophysics, Cambridge, MA, USA \\ ${ }^{d}$ Center for General Education, Kagoshima University, Kagoshima, Japan \\ E-mail: nadyaeasc.rssi.ru
}

\begin{abstract}
$\mathrm{H}_{2} \mathrm{O}$ masers associated with the massive star formation region $\mathrm{W} 49 \mathrm{~N}$ were observed in frames of the Space-VLBI project RadioAstron. Ultra-compact structures in the masers were detected on space-ground baselines up to 9.6 Earth diameters. The flux density of ultra-compact features does not exceed a percent of the single dish flux density. Some results of the data processing for three observing sessions performed in 2014-2015 are presented. The lower limit of minimal brightness temperatures and estimates of angular sizes of the brightest features corresponding to the ultra-compact maser spots of the W49N complex are obtained.
\end{abstract}

14th European VLBI Network Symposium \& Users Meeting (EVN 2018)

8-11 October 2018

Granada, Spain

${ }^{*}$ Speaker. 
Table 1: Observing sessions summary.

\begin{tabular}{clcccc}
\hline \hline Epoch & Ground telescope array & $\begin{array}{c}\text { Observing } \\
\text { time }\end{array}$ & Band & $\begin{array}{c}\text { Baseline } \\
\text { length }\end{array}$ & $\begin{array}{c}\text { Fringe } \\
\text { spacing }\end{array}$ \\
\hline 2014 May 18 & Effelsberg 100-m & 1 hour & $22 \mathrm{GHz}$ & $\begin{array}{c}3.0 \mathrm{ED} \\
\sim 38000 \mathrm{~km}\end{array}$ & $74 \mu$ as \\
& & 1 hour & $22 \mathrm{GHz}$ & $\begin{array}{c}9.6 \mathrm{ED} \\
22\end{array}$ & 23 as \\
\hline 2015 April 27 & Effelsberg 100-m & & & & \\
& Yebes 40-m & & & & \\
& Torun 32-m & & & & \\
& Hartebeesthoek 26-m & & & & \\
\hline 2015 May 22 & Green Bank 100-m & 50 min & $22 \mathrm{GHz}$ & $8.6 \mathrm{ED}$ & 26 as \\
& Effelsberg 100-m & & & $\sim 110000 \mathrm{~km}$ & \\
& Medicina 32-m & & & & \\
& Torun 32-m & & & & \\
\hline \hline
\end{tabular}

\section{Introduction}

The high-mass star-forming region $\mathrm{W} 49 \mathrm{~N}(\mathrm{G} 43.16+0.1)$ is a part of W49A, which is the most massive and luminous star-forming complex in our Galaxy. The region W49N is located in the Perseus arm near the solar circle in the first Galactic quadrant at the distance from the Sun of $11.11 \pm 0.8 \mathrm{kpc}$ [1]. The distance to $\mathrm{W} 49 \mathrm{~N}$ from the Galactic mid-plane is only $3 \mathrm{pc}$. This region contains numerous $22 \mathrm{GHz} \mathrm{H}_{2} \mathrm{O}$ masers forming the most luminous maser set $\left(\sim 1 \mathrm{~L}_{\odot}\right)$ in the Galaxy. This makes the W49N region an excellent target for RadioAstron maser observations.

RadioAstron (RA) is the international space VLBI project involving the 10-m Space Radio Telescope (SRT) on board the satellite Spektr-R and ground radio telescopes [2]. The SRT was launched in 2011 on elliptical orbit whose plane is evolving with time with an apogee of up to $370000 \mathrm{~km}$ and operates at frequencies of $22,5,1.6$, and $0.3 \mathrm{GHz}$ [3]. W49N was observed as part of the RadioAstron maser survey during 2014-2015 [4]. The highest angular resolution of $23 \mu$ as for galactic masers was achieved in W49N observations on a baseline of 9.6 Earth diameters (ED).

\section{Observations}

We conducted three observing sessions of W49N as listed in the Table 1. Left- and right-hand circular polarization data were recorded with a total bandwidth of $32 \mathrm{MHz}$ per polarization. Crosscorrelation data were obtained in a frequency channel spacing of $7.81 \mathrm{kHz}$. The antenna pointing and phase-tracking center for all W49N observations in Table 1 was set to RA $=19^{h} 10^{m} 13.4096^{s}$, $\mathrm{DEC}=09^{\circ} 06^{\prime} 12.803^{\prime \prime}$, where the most luminous UCHII region $\mathrm{G}$ in the W49N complex is located.

\section{Results}

The $\mathrm{H}_{2} \mathrm{O}$ maser in $\mathrm{W} 49 \mathrm{~N}$ is known as a highly variable source demonstrating high-velocity bright features arising from time to time in a range of several hundred $\mathrm{km} / \mathrm{s}$. Such features may have flux densities up to several thousand Jy and then disappear. The main part of the W49N $\mathrm{H}_{2} \mathrm{O}$ maser spectrum around the systemic velocity at $\sim 0 \mathrm{~km} / \mathrm{s}$ was always observable and had a stably high flux density. Since RadioAstron can observe in the bandwidth of $32 \mathrm{MHz}$, the central frequency in all observations of $\mathrm{W} 49 \mathrm{~N}$ was chosen so that the main part of the spectrum fell into the 

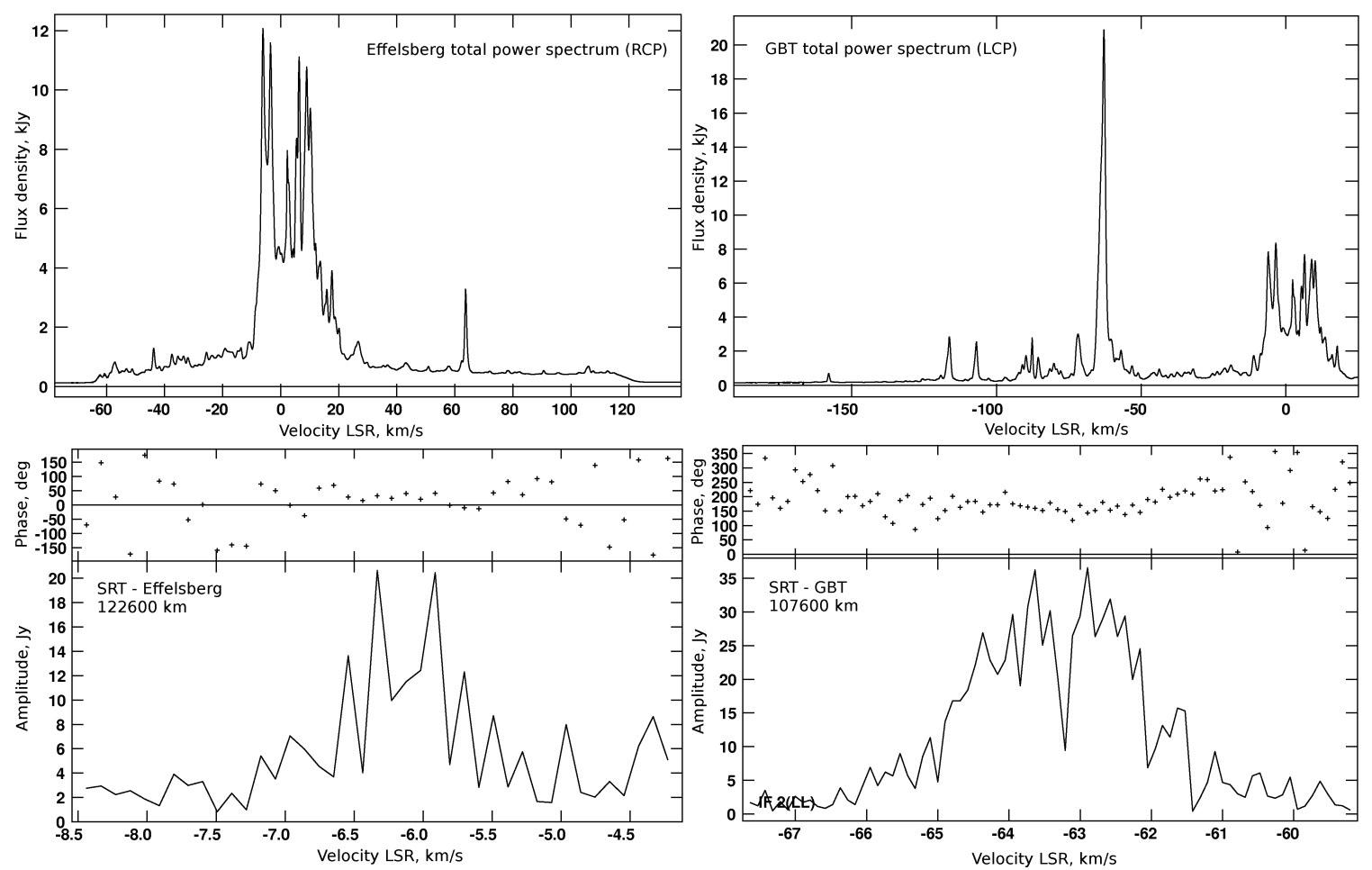

Figure 1: Top panel: Autocorrelation spectra of W49N obtained in the RadioAstron observations on April 27, 2015 (left) and May 22, 2015 (right). Telescopes are indicated on the figures. $\mathrm{V}_{L S R}$ ranges correspond to the upper side band used in these observing sessions with central frequency of $22228 \mathrm{MHz}$ and $22236 \mathrm{MHz}$ respectively. Bottom panel: Cross-correlation spectra of W49N maser features detected on space-ground baselines obtained in the RadioAstron observations. Corresponding length of baselines and telescopes are indicated on the figures.

band. Nevertheless, during the observing session on 27 April 2015 a short-living feature at $64 \mathrm{~km} / \mathrm{s}$ was observed (see Figure 1, top left panel). According to monitoring on the Effelsberg 100-m radio telescope [5], this feature appeared a few weeks before the observation on RadioAstron and disappeared in a few months later. During the observation on 22 May 2015 a bright feature at $-63 \mathrm{~km} / \mathrm{s}$ was observed (see Figure 1, top right panel). The results of the post correlation data processing for these two sessions are shown on Figure 1. Amplitude calibration of the data was carried out using $\mathrm{T}_{\text {sys }}$ measurements obtained during observations.

Fringes on a space baseline of $\sim 3$ ED between RA and 100-m RT in Effelsberg were found in the first session on May 18, 2014 for two spectral features at $-6 \mathrm{~km} / \mathrm{s}$ and at $6 \mathrm{~km} / \mathrm{s}$ (see Figure 2). Lack of the intermediate length baselines does not allow us to explore the structure of the features so as to distinguish the flux density contributions from different structural components. With a given angular resolution we can obtain only a lower limit of the brightness temperature of a component as was suggested in [6]. Thus, for the brightest feature at $-6 \mathrm{~km} / \mathrm{s}$ we obtained $\mathrm{T}_{b, \min } \sim 6.1 \mathrm{e}+14 \mathrm{~K}$.

In the second session on April 27, 2015, the brightest feature at the velocity $-6 \mathrm{~km} / \mathrm{s}$ was detected at the RA-Effelsberg baseline at the fringe spacing $23 \mu$ as, what is the record fringe spacing achieved for galactic masers (see Figure 1, bottom left panel). The minimal brightness temperature of the corresponding maser spot is $\mathrm{T}_{b, \min } \sim 5.7 \mathrm{e}+14 \mathrm{~K}$. The short-living feature at 
$64 \mathrm{~km} / \mathrm{s}$ has a fringe only on ground baselines up to the longest baseline Effelsberg-Hartebeesthoek.

In the third session on May 22, 2015, fringes at the space baseline RA-GBT were found for a group of features at VLSR $-63 \mathrm{~km} / \mathrm{s}$ (see Figure 1, bottom right panel). Weak fringe was also found for feature at $-72 \mathrm{~km} / \mathrm{s}$. The minimal brightness temperature of the brightest feature at $-63 \mathrm{~km} / \mathrm{s}$ is $\mathrm{T}_{b} \sim 1.1 \mathrm{e}+15 \mathrm{~K}$.

It is quite interesting that the three brightest components detected at space baselines in three experiments contain only $0.006,0.001$ and 0.002 of the total flux densities, respectively. Fringe spacings given in the last column of the Table 1 can be considered as nominal estimates of actual sizes of these components. Another thing, which should be taken into account, is that we could see the refractive scattering floor caused by interstellar scintillation when observing at such long baselines up to $9.6 \mathrm{ED}[7]$.
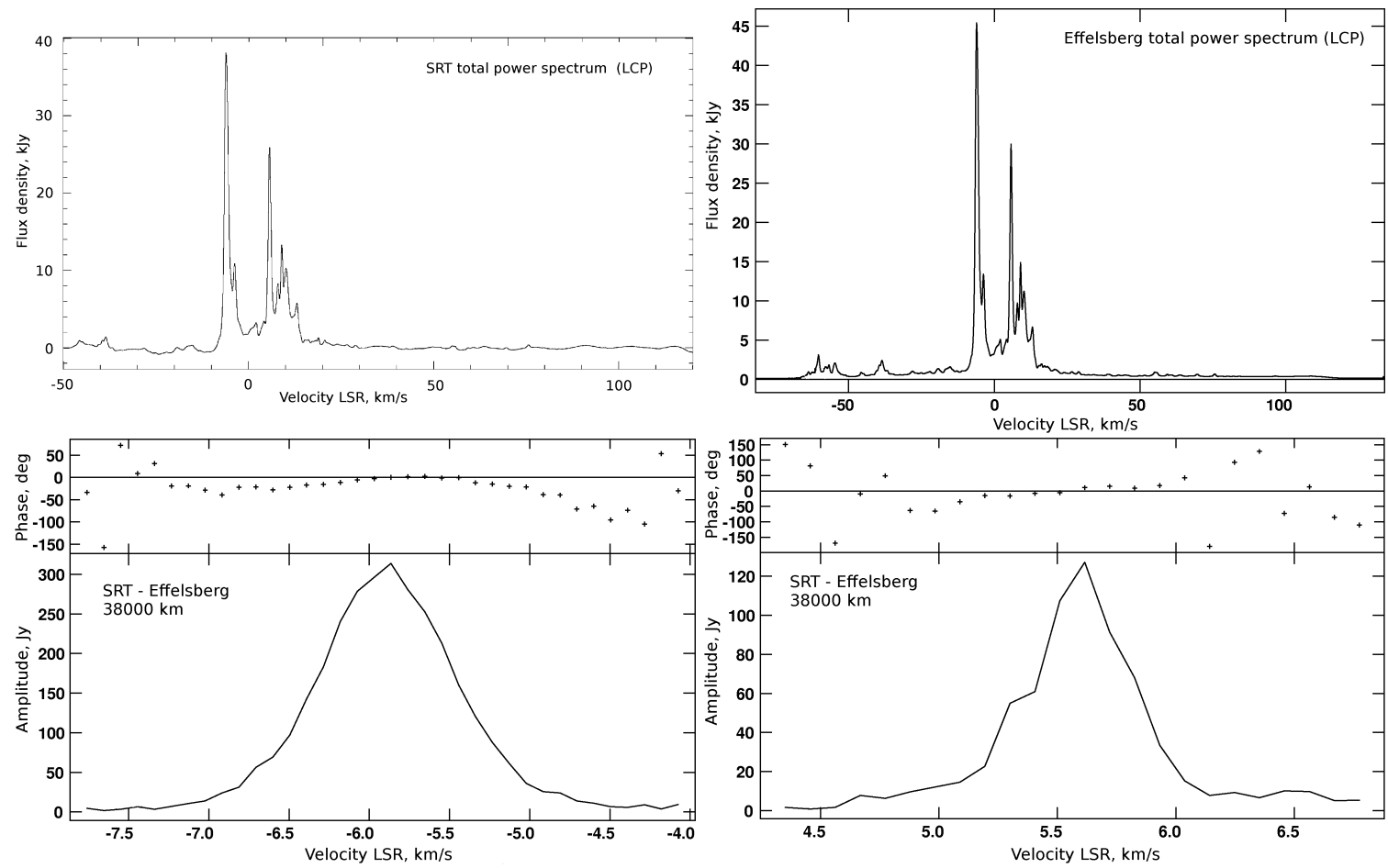

Figure 2: Top panel: Autocorrelation spectra of W49N obtained on the 10-m SRT (left) and 100-m Effelsberg (right) in the RadioAstron observation on May 18, 2014. $\mathrm{V}_{L S R}$ range at Effelsberg spectrum corresponds to the upper side band used in this observing session with central frequency of $22228 \mathrm{MHz}$. Bottom panel: Cross-correlation spectra of two W49N maser features detected on space-ground baseline between SRT and Effelsberg. Telescopes and corresponding length of baseline are indicated on the figures.

\section{References}

[1] B. Zhang, M. J. Reid, K. M. Menten, X. W. Zheng, A. Brunthaler, T. M. Dame, and Y. Xu, Parallaxes for W49N and G048.60+0.02: Distant Star Forming Regions in the Perseus Spiral Arm, The Astrophysical Journal, 775, 79 (2013).

[2] N. S. Kardashev, V. V. Khartov, V. V. Abramov, V. Yu. Avdeev, A. V. Alakoz et al., RadioAstron - a Telescope with a Size of 300000 km: Main Parameters and First Observational Results, Astronomy Reports, 57, 153 (2013). 
[3] http://www.asc.rssi.ru/radioastron/index.html

[4] A. M. Sobolev, N. N. Shakhvorostova, A. V. Alakoz, W. A. Baan, RadioAstron Maser Observations: a Record in Angular Resolution, Proceedings of the conference "Stars: From Collapse to Collapse", Astronomical Society of the Pacific, 510, 27 (2017).

[5] B. Hutawarakorn Kramer, Variability of Water Masers in W49N: Results from the Effelsberg Long-term Monitoring Program (2014-2017), Effelsberg Science Workshop 2018, Bonn, 20 Febrary (2018).

[6] A. Lobanov, Brightness temperature constraints from interferometric visibilities, Astronomy \& Astrophysics, 574, A84 (2015).

[7] M. D. Johnson, Y. Y. Kovalev, C. R. Gwinn, L. I. Gurvits et al., Extreme Brightness Temperatures and Refractive Substructure in 3C273 with RadioAstron, The Astrophysical Journal Letters, 820, 1, L10, 6 (2016). 